\title{
Anti-Müllerian hormone: a critical factor for female fertility and reproductive health
}

\author{
Marzena Rzeszowska ${ }^{1}$, Agnieszka Leszcz ${ }^{1}$, Lechosław Putowski ${ }^{1,2}$, Magdalena Hałabiśs ${ }^{3}$, \\ Joanna Tkaczuk-Włach ${ }^{1,2}$, Jan Kotarski ${ }^{4}$, Grzegorz Polak ${ }^{1,4}$ \\ ${ }^{1}$ A b ovo Sp. z o.o., Family Health Center, Lublin, Poland \\ ${ }^{2}$ Departament of Gynecology and Gynecological Endocrinology, Medical University of Lublin, Poland \\ ${ }^{3}$ Departament of Laboratory Diagnostics, Medical University of Lublin, Poland \\ ${ }^{4} 1^{\text {st }}$ Department of Gynecological Oncology and Gynecology, Medical University of Lublin, Poland
}

\begin{abstract}
Anti-Müllerian hormone (AMH) is a glycoprotein produced by the granulosa cells of preantral and small antral follicles. AMH concentrations reflect ovarian physiology with high precision, thus serving as a more sensitive marker of the ovarian reserve than chronological age. This hormone plays a role in the pathogenesis of menstrual disorders and fertility in both obesity and polycystic ovary syndrome. The evaluation of AMH may also be useful in diagnosing or monitoring therapy of granulosa cell ovarian tumors.
\end{abstract}

Key words: anti-Müllerian hormone, fertility, gynecology

\section{INTRODUCTION}

Anti-Müllerian hormone (AMH), discovered in the 1950s by Alfred Jost and colleagues, is an important factor involved in differentiation of the internal sex organs of the embryo [1]. Expression of the AMH gene, also known as MIS (Müllerian Inhibitor Substance), results in the loss of Müller ducts during sexual differentiation to the male gender [2]. In women, AMH is produced by granulosa cells in primordial preantral and small antral follicles and participates in folliculogenesis regulation. This occurs by inhibiting the germinal vesicle recruitment process through reduction of FSH's effect on follicular growth of preantral and antral follicles.

The recent creation of commercially available diagnostic tests for measuring serum AMH concentration has allowed for wide-scale research studies focused on utilzing $\mathrm{AMH}$ as a clinical marker of the ovarian reserve. Additionally, it can also be used as a predictive factor with regards to ovarian stimulation with gonadotropins during ovulation stimulation.
The evaluation of $\mathrm{AMH}$ in women participating in the program "Treatment of infertility by in vitro fertilization", provided by the Ministry of Health, has become one of the selection criteria for assisted reproduction procedures.

This work summarizes the current knowledge about the role and use of $\mathrm{AMH}$ in gynecological practices.

\section{ANTI-MÜLLERIAN HORMONE STRUCTURE}

The anti-Müllerian hormone is a glycoprotein with a molecular weight of $140 \mathrm{kDa}$, belonging to the TGF- $\beta$ growth factor superfamily. The human AMH gene is located on the short arm of chromosome 19 and is comprised of 275 base pairs, which are divided into five exons. Its gene product is a 560 amino acid precursor, proAMH. 24 amino acids consisting fragment is removed from proAMH than the molecule is glycosylated. In the next step two identical proAMH subunits with a molecular weight of $70 \mathrm{kDa}$ linked to each other by sulfide bridges. ProAMH proteolysis leads to the formation of the N-terminal fragment called the "pro-region" (115 kDa 

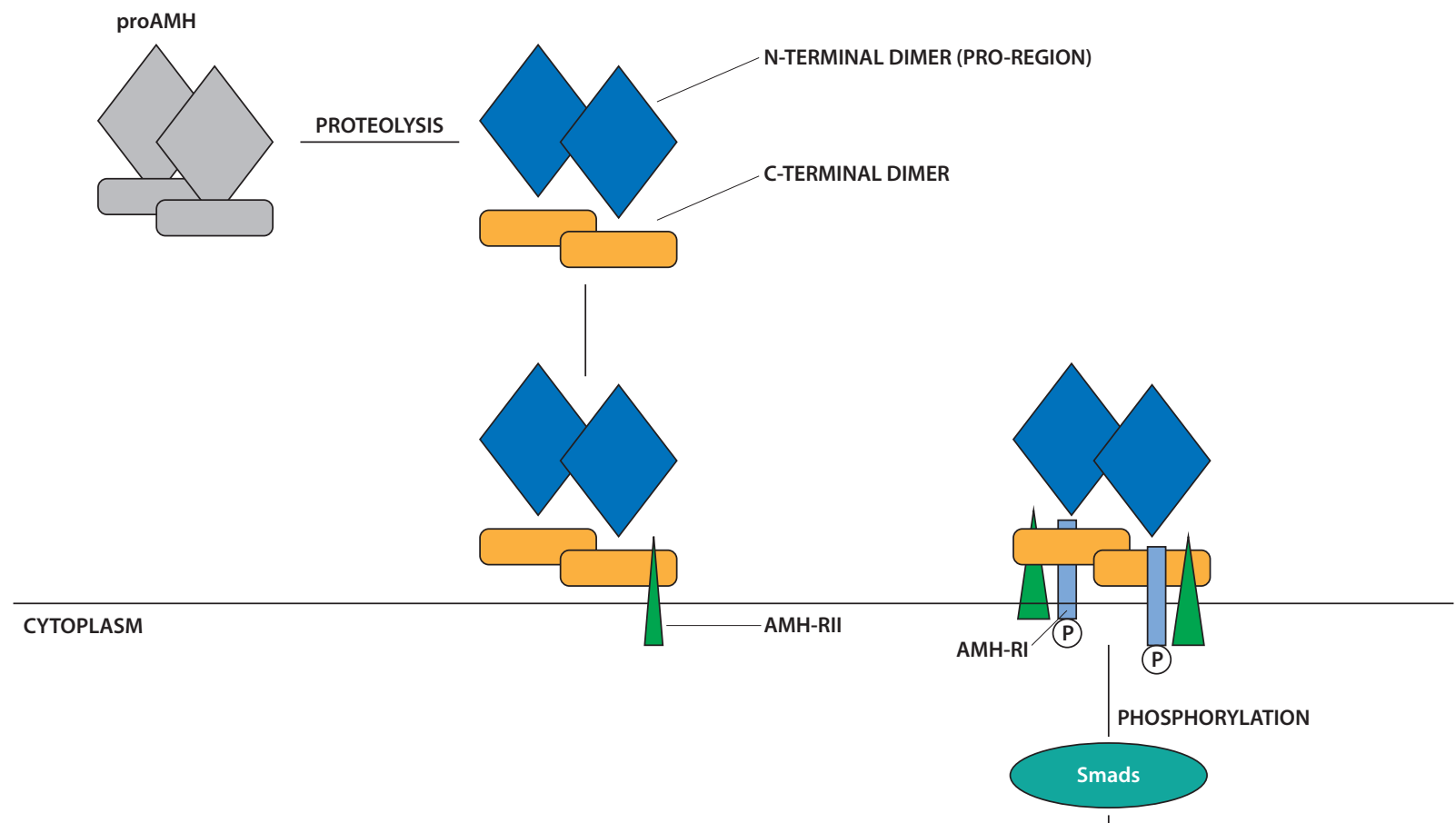

NUCLEUS

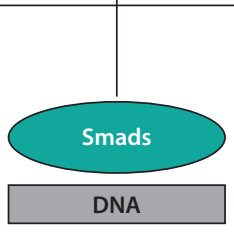

Figure 1. The mechanism of $\mathrm{AMH}$ action via receptor Proteolysis of $\mathrm{AMH}$ pro leads to a conformational change in the $\mathrm{C}$-terminal domain that allows binding AMRH II. AMH-RII induces binding type I receptor, which is phosphorylated by a kinase receptor type II. The activated AMH-RI in turn phosphorylates further Smads proteins. Phosphorylated Smads specific receptors translocate to the nucleus, where they regulate gene expression

AMHN), and a C-terminal domain, i.e. "mature region" $25 \mathrm{kDa}$ $\mathrm{AMHC}$ ). The C-terminal fragment is responsible for the biological activity of the protein and receptor binding, but in order to be active, it requires the $\mathrm{N}$-terminal fragment unlike other proteins in the TGF- $b$ family [3].

AMH acts on two types of receptors: $\mathrm{AMH}-\mathrm{I}$ and $\mathrm{AMH}-\mathrm{II}$, both present in mesenchymal cells. AMH does not have direct affinity for $\mathrm{AMH}-\mathrm{I}$; however, it binds to the $\mathrm{AMH}-\mathrm{II}$. This allows for the connection of receptor I and the formation of a large complex consisting of the $\mathrm{AMH}$ protein dimer, and two particles of both AMH-RI and AMH-RII. This combination induces tyrosine phosphorylation of $\mathrm{AMH}-\mathrm{RI}$, leading to further reactions in which the Smad protein complex (proteins involved in the signal transduction into the cell following TGF- $\beta$ activation) is transported to the nucleus where it regulates gene transcription (Figure 1) $[4,5]$.

\section{AMH AND FOLLICULOGENESIS}

In females, fetal expression of $\mathrm{AMH}$ is observed from the $36^{\text {th }}$ week of pregnancy. In women, AMH is produced by granulosa cells in primordial preantral and small antral follicles. The production of $\mathrm{AMH}$ in the follicle starts from the moment of its recruitment and lasts to antral stage development. The highest level of hormone synthesis is observed in granulosa cells of preantral and small antral follicles (to $4 \mathrm{~mm}$ ). In larger antral follicles (> $8 \mathrm{~mm}$ ), AMH synthesis slowly decreases until it becomes undetectable [6].

$\mathrm{AMH}$ inhibits gene and protein lexpression of cytochrome P450 in aromatase granular cells [7]. By reducing the effect of FSH on preantral and antral follicle growth, the anti-Müllerian hormone participates in the regulation of folliculogenesis, thereby inhibiting the recruitment of germinal vesicles.

Knowledge concerning the effect of $\mathrm{AMH}$ on folliculogenesis was discovered using mice lacking the AMH (type null). The ovaries of four-month-old null mice were twice as large as a wild type mouse, and they contained a lower number of germinal vesicles and a three-fold increase in small growing vesicles [5]. This was due to the increased recruitment of primordial follicles and their transformation to preantral and antral follicles.

Null mice with the larger number of follicles also showed lower levels of FSH, which allowed for the hypothesis that, in the absence of $\mathrm{AMH}$, follicles are more sensitive to the 
influence of FSH. In in vitro mouse studies, $\mathrm{AMH}$ addition to follicular culture inhibited FSH-dependent follicular growth, resulting in a smaller follicle diameter [8]. In vivo studies on null mice showed greater follicle growth occurred at low and at high exogenous FSH administration as compared to wild type animals. The inhibitory effect of AMH on FSH follicular sensitivity may occur during the follicle selection process [5].

Current theories suggest that AMH acts as a co-regulator of steroidogenesis in granulosa cells. As it has been shown, the $\mathrm{AMH}$ concentraiton correlates with estradiol levels in small antral follicle fluid. Studies have shown a relationship between an $\mathrm{AMH}$ gene polymorphism or the $\mathrm{AMH}$ receptor type Il and the level of estradiol in the follicular phase, pointing to a role for $\mathrm{AMH}$ in $\mathrm{FSH}$-dependent steroidogenesis in the human ovary [9].

AMH is detected in the serum, although its main role is auto and paracrine action on follicular development. Antral follicles appear to be a major source of AMH in the serum because of the larger number of granulosa cells. The presence of AMH in the blood stream has been the subject of many studies that are focused on the possibility of the use of hormone evaluation in the diagnosis of endocrine and oncological disorders.

\section{AMH AS A MARKER OF FEMALE FERTILITY}

During a woman's life, serum AMH concentrations in serum are low after birth and during the prepubertal phase. At puberty, AMH concentration the in blood increases, peaking at approximately 20-25 years of age. After this time, hormone concentrations decreases to undetectable levels following menopause [10].

$\mathrm{AMH}$ appears to be a good marker in the assessment of the ovarian reserve. In women, AMH levels decrease with age before signs of upcoming menopause manifest, such as an increase in FSH concentration or a decrease in the number of antral follicles. During menopause, AMH becomes undetectable, a phenomena associated with the depletion of the follicular reserve. Thus, the reduced concentration of serum $\mathrm{AMH}$ may indicate the physiological or pathological aging of the ovary [11].

During one menstrual cycle, hormone levels are not significantly affected $[12,11]$, although Wunder et al. showed lower concentrations of AMH in the early luteal phase [13]. The described variation in the cycle is not significant enough to recommend $\mathrm{AMH}$ measurements on specific days of the menstrual cycle.

Various factors have been reported to influence differences in AMH concentrations: ethnicity, smoking, vitamin D levels, and obesity. It was found that African-American and Spanish women have lower values of AMH than Caucasian women. Also, women who smoke present a lower level of anti-Müllerian hormone than non-smokers [14]. Sowers et al. has shown that, in smokers, AMH concentrations decrease faster, and these women reach the age of menopause earlier than non-smokers [15]. A positive correlation between serum 25-hydroxy vitamin $\mathrm{D}$ and serum $\mathrm{AMH}$ has been shown, which may be related to the presence of a binding complex for vitamin $D$ in the promoter of the AMH gene [13]. It has been noted that the concentration of $\mathrm{AMH}$ and inhibin $\mathrm{B}$ is lower in women with a high BMI but without Polycystic Ovary Syndrome (PCOS) [16]. In the study of obese women compared to those of normal weight, a decreased level of $\mathrm{AMH}$ was observed; however, there were no differences between the amount of antral follicles in the USG image [17]. Nevertheless, the effectiveness of in vitro fertilization (IVF) programs in obese women is also smaller. The correlation mechanisms between obesity and ovarian function with respect to $\mathrm{AMH}$ production are not fully understood. One mechanism may include a lipotoxicity effect on granular cells. In a mouse study in which animals were treated with a high-fat diet, a higher percent of anovulation cycles was observed. Additionally, decreased fertility, lipid accumulation, endoplasmic reticulum stress, mitochondrial dysfunction, and apoptosis of granular cells and the crown were noted [18].

It has been observed that in women with PCOS syndrome (Polycystic Ovary Syndrome), the level of AMH is 2-3 times higher than in healthy women with normal ovulation. A growing number of studies suggest that the diagnosis of PCOS, which is now determined using an ultrasound evaluation of the ovaries, anovulatory cycles, and hyperandrogenemia, should be replaced by the measurement of $\mathrm{AMH}$. Carrying out a meta-analysis of anti-Müllerian hormone concentrations in women with PCOS (AMH cut-off of $4.7 \mathrm{ng} / \mathrm{mL}$ ), the obtained sensitivity and specificity were $82.8 \%$ and $79.4 \%$, respectively [12]. Histological examination of the ovaries of women with PCOS showed a normal number of primordial follicles, and a two-fold increase in the number of growing follicles. The elevated level of $\mathrm{AMH}$ is connected with a larger number of preantral and small antral follicles, which are the major source of the hormone. The high level of AMH is responsible for follicle growth inhibition during the selection process of a dominant follicle through the negative correlation of $\mathrm{AMH}$ and $\mathrm{FSH}$, thereby leading to oligoovulation. Anti-Müllerian hormone inhibits aromatase synthesis, which is activated by the action of $\mathrm{FSH}$ on granular cells. Higher concentrations of $\mathrm{AMH}$ are observed in patients with insulin resistance as compared to patients with normal insulin sensitivity [7]. Similarly, women with anovulation have a higher level of AMH than those who are oligoovulatory, which may point to a role of $\mathrm{AMH}$ in PCOS pathogenesis in patients with anovulatory cycles. High concentrations of $\mathrm{AMH}$ have also been demonstrated in adolescent girls (12-18 years) with PCOS [19], as well as in 
4-7-year-old daughters of women suffering from this disease. The AMH concentration in women with PCOS declines with age, but it clearly occurs after the $40^{\text {th }}$ decade of life.

\section{THE USE OF AMH IN ASSISTED REPRODUCTION TECHNIQUES}

The level of AMH slowly drops after gonadotropin treatment during controlled ovarian stimulation [20]. This decrease could be an effect of a direct or indirect negative influence of FSH on AMH ovarian secretion. The exogenous FSH treatment elevates estradiol, which could be the source of $\mathrm{AMH}$ decrease as estradiol negatively influences the regulation of AMH and AMHII mRNA in the ovary [21].

Studies have reported a positive correlation between serum AMH levels and the number of obtained eggs [20]. It appears that the pretreatment level of $\mathrm{AMH}$ is a better prognostic marker than patient age, the level of FSH on the $3^{\text {rd }}$ day of the cycle, and the levels of estradiol or inhibin B. A number of antral follicles (AFC-antral follicle count) in the USG image were positively correlated with $\mathrm{AMH}$ concentration. Low levels of anti-Müllerian hormone and AFC are weak prognostic markers for stimulation in in vitro fertilization (IVF), and they are considered to be equivalent parameters for prediction of treatment success. The risk of IVF cycle cancellation could be 13 times higher in patients with undetectable AMH levels than in those with an AMH level higher than $2.0 \mathrm{ng} / \mathrm{mL}$ [22]. Decreased AMH concentrations do not exclude the possibility of getting pregnant, although the success rate is much lower. In a study by Kedema et al. [23], conducted in 769 IVF/ICSI cycles, the rate of achieved pregnancies was the same and equal to $4.4 \%$ in two studied groups. In one group of patients the level of AMH was low $(0,2-1,0 \mathrm{ng} / \mathrm{mL})$ in second group extremely low $(<0.2 \mathrm{ng} / \mathrm{mL})$. In women older than 42 years of age, with AMH concentrations $<0.2 \mathrm{ng} / \mathrm{mL}$, pregnancy was not achieved. However, analysis presented by Łukaszuk et al. [24] examined 101 women with $\mathrm{AMH}$ levels $<0.4 \mathrm{ng} / \mathrm{mL}$ during 188 in vitro cycles and achieved 14 live births, making the IVF procedure $7.45 \%$ effective. Thus, low concentrations of AMH require different stimulation protocols, in particular, short and ultrashort protocols with high initial dosages of gonadotropins [22].

The evaluation of anti-Müllerian hormone is an important predictor factor of ovarian hyperstimulation syndrome (OHSS) risk. AMH is a better prediction marker for an excessive ovarian response due to its stimulation as opposed to gonadotropin level, age, or BMI. It is also more precise than follicle number in the USG image or the estradiol level after HCG administration. A value $3.5 \mathrm{ng} / \mathrm{mL}$ is considered the cut-off point, indicating a high risk of OHSS. During the cycles of in vitro fertilization, it is recommended to use low initial doses of gonadotropins and protocols with a $\mathrm{GnRH}$ antagonist [20].
In a prospective study, Nelson et al. showed a higher rate of live births is associated with higher concentrations of AMH. However, the authors suggest that such a good correlation may be related to a greater number of oocytes [25]. Other randomized studies conducted by Brodin et al. [26] on 842 women confirmed a strong positive correlation between the serum AMH levels and the amount of received oocytes, the rate of achieved pregnancies, as well as a cumulative indicator of live births.

It seems that AMH is a good marker for quantitative, rather than qualitative, techniques of assisted reproduction. The individual selection of the type of stimulation protocol and doses of gonadotropins in IVF programs, based on the concentration of $\mathrm{AMH}$, allows for a safer and more effective use of in vitro fertilization.

\section{AMH AS A CANCER MARKER}

The expression of AMH by granulosa cells of ovarian small antral and primordial follicles attempted to use AMH as a marker of cancer derived from granular cells (GTCs, granulosa cell tumors). These tumors comprise about $3 \%$ to $5 \%$ of all ovarian tumors. It was noted that $\mathrm{AMH}$ concentration was elevated in $76-93 \%$ of women with tumor cells derived from granular cells [27]. Furthermore, higher concentrations of $\mathrm{AMH}$ were observed up to 16 months before tumor relapse. Therefore, the determination of the hormone value is a useful marker in detecting recurrences of GTCs, in particular by using new generation ultrasensitive assays.

Recent studies indicate that a large number of primary epithelial ovarian tumors originate from the scrap of the fallopian tubes or the components of the secondary Müller system. Based on the fact that AMH is responsible for the regression of the Müllerian ducts, there has been an attempt to use this hormone in the treatment of epithelial ovarian cancers. Growth inhibition in epithelial tumor cell lines after incubation with the AMH was achieved in in vitro studies [28].

Chemotherapy is particularly toxic for the ovaries by promoting follicle injury. It has been shown that, after chemotherapy, AMH concentration is significantly lower than in healthy women at a similar age, which confirms the idea that the hormone is a sensitive indicator of ovarian reserve. Depending on the drugs and doses used in therapy, $\mathrm{AMH}$ levels after treatment may vary. Hormone levels were shown to be significantly lower or almost undetectable after the therapy with alkylating agents in comparison to other types of chemotherapy. The elevation of AMH after chemotherapy depends on the type of the treatment protocol [29].

AMH was identified as a marker used to classify patients for fertility preservation procedures before anticancer therapy. Therefore, it is important to direct women to infertility treatment facilities as soon as possible to select the appropriate treatment options such as cryopreservation 
of the obtained embryos, oocytes, or freezing ovarian tissue for the afterward transplant.

\section{AMH MEASUREMENT}

Initial reports determining anti-Müllerian hormone concentrations first emerged in the 90's of XX century. The first generation, commercially available tests were produced by Diagnostics Systems Ltd. (DSL) and Immunotech Ltd. (IOT). Each test contained different types of antibodies and standards, a reason for differences in reported AMH levels [30]. Subsequent analysis showed similarity in AMH levels, potentially a result of kit improvement. The consolidation of the two companies by Beckman Coulter resulted in the introduction of one test, AMH Gen II Assay, which contained antibodies from the DSL kit and standards from IOT kit. The $\mathrm{AMH}$ results obtained with using the second-generation test were about $22-40 \%$ higher than the first generation test results; however, they corresponded with each other [31]. Further studies discredit the serum sample stability during storage time, dilution, and even AMH measurement [32]. Rustamov et al. noted that the level of $\mathrm{AMH}$ obtained with the AMH Gen II kit was approximately $20 \%$ lower than the results obtained by the DSL kit. The decrease in quality of the second-generation tests (also those produced since the year 2012, AMH Gen II kit with IVD certificate) was demonstrated during an analysis conducted in 8,323 samples. Levels of AMH obtained by the second-generation test, Gen II IVD, were $40 \%$ lower than the previous generation of Gen II kit and $70 \%$ lower than the IOT kit [33]. In response to reports regarding test accuracy, Beckman Coulter introduced a new protocol for $\mathrm{AMH}$ evaluation with the recommendation of serum pre-incubation with a suitable buffer (Conference ESHRE, London 2013) prior to measurement. The dilution of the sample was to prevent errors resulting from the effect of complement protein binding. Additionally, to improve sample stability, it was reccommended to freeze the serum within two hours after its collection.

The newest generation tests like Ultrasensitive (2012) and PicoAMH (2013), produced by AnsLabs, show a higher sensitivity and detection threshold than AMH Gen II IVD ( 0.023 vs. 0.001 vs. $0.08 \mathrm{ng} / \mathrm{mL}$, respectively), and they could be more useful in detecting low levels of anti-Müllerian hormone [34]. Published data has pointed out the necessity of a cautious interpretation of results obtained using the $\mathrm{AMH}$ Gen II kit. For that reason, it seems to be rather impossible to compare the results, cut-off points, or clinically important values obtained with the test produced by different manufacturers.

The introduction of the first automatic AMH test in September 2014 by Elecsys (ECLIA method) Roche could be the next step in the creation of a test appropriate in clinical practice. The manufacturer assigned individual reference ranges for women at different ages and defined the cut-off value for $\mathrm{AMH}$ in patients with PCOS [35]. The determination of an international AMH standard could be an important step toward the creation of a test completely useful in clinical practice.

\section{SUMMARY}

Anti-Müllerian hormone reflects ovarian function with high precision. AMH is a more sensitive marker of ovarian reserve than chronological age, and, for this reason, it seems very helpful in reproductive planning for women using both natural and assisted reproductive technologies. It is also one of the elements of pathogenesis of menstrual disorders and fertility in obese women in cases with and without PCOS coexistence. The evaluation of AMH may be very useful in diagnosing or monitoring the treatment of ovarian cancer derived from granulosa cells.

\section{REFERENCES}

1. Josso N. Professor Alfred Jost:The Builder of Modern Sex Differentiation. Sex Dev. 2008, 2, 55-63.

2. Charon KM, Świtoński M. Genetyka zwierząt. Warszawa. PWN, 2004.

3. Pepinsky RB, Sinclair LK, Chow EP. Proteolytic processing of mullerian inhibiting substance produces a transforming growth factor- $\beta$-like fragment. J Biol Chem. 1988, 263, 18961-18964.

4. Skałba P, Cygal A, Dąbkowska-Huć A. Wpływ hormonu anty-Mullerowskiego (AMH) na folikulogeneze. Ginekol Pol. 2008, 79, 137-140.

5. Durlinger A, Kramer $P$, Karels $B$, [et al.]. Control of primordial follicle recruitment by anti-Müllerian hormone in the mouse ovary. Endocrinol. 1999, 140, 5789-5796.

6. Jeppesen JV, Anderson RA, Kelsey TW, [et al.] Which follicles make the most anti-Mullerian hormone in humans? Evidence for an abrupt decline in AMH production at the time of follicle selection. Mol Hum Reprod. 2013, 19, 519-527

7. Grossman M, Nakaji S, Fallat M, [et al.]. Müllerian inhibiting substance inhibits cytochromeP450 aromataze activity in human granulosa lutein cell culture. Fertil Steril. 2008, 89, 1364-1370.

8. Durlinger A, Gruijters M, Kramer P, [et al.]. Anti-Müllerian hormone attenuates the effect of FSH on follicle development in the mouse ovary. Endocrinol. 2001, 142, 4891-4899.

9. Kevenaar ME, Themmen AP, Laven JS, [et al.]. Anti-Müllerian hormone and anti-Müllerian hormone type II receptor polymorphisms are associated with follicular phase estradiol levels in normo-ovulatory women. Hum Reprod. 2007, 22, 1547-1554.

10. Lie Fong $\mathrm{S}$, Visser JA, Welt CK, [et al.]. Serum anti-müllerian hormone levels in healthy females: a nomogram ranging from infancy to adulthood. J Clin Endocrinol Metab. 2012, 97, 4650-4655.

11. De Vet A, Loven J, de Jong F, [et al.]. Anti-Müllerian hormone serum levels: a putative marker for ovarian aging. Fertil Steril. 2002, 77, 357-362.

12. Iliodromiti S, Kelsey TW, Anderson RA, [et al.]. Can anti-Mullerian hormone predict the diagnosis of polycystic ovary syndrome? A systematic review and meta-analysis of extracted data. J Clin Endocrinol Metab. 2013, 98, 3332-3340.

13. Wunder DM, Bersinger NA, Yared M, [et al.]. Statistically significant changes of antimüllerian hormone and inhibin levels during the physiologic menstrual cycle in reproductive age women. Fertil Steril. 2008, 89, 927-933.

14. Tal R, Seifer DB. Potential mechanisms for racial and ethnic differences in antimüllerian hormone and ovarian reserve. Int JEndocrinol. 2013: 818912.

15. Sowers MR, McConnell D, Yosef M, [et al.]. Relating smoking, obesity, insulin resistance and ovarian biomarker changes to the final menstrual period (FMP) Ann N Y Acad Sci. 2010, 1204, 95-103.

16. Freeman E, Gracia C, Sammel M, [et al.]. Association of anti-Müllerian hormone levels with obesity in late reproductive age woman. Feril Steril. 2007, 87, 101-106.

17. Su HI, Sammel M, Freeman E, [et al.]. Body size affects measures of ovarian reserve in late reproductive age women. Menopause. 2008, $15,857-861$. 
18. Wu LL-Y, Dunning KR, Yang X, [et al.]. Hight-fat diet causes lipotoxicity responses in cumulus- oocyte complexes and decreased fertilization rates. Endocrinol. 2010, 151, 5438-5445.

19. Siow $Y$, Kives $S$, Hertweck $P$, [et al.]. Serum Müllerian-inhibiting substance levels in adolescent girls with normal menstrual cycles or with polycystic ovary syndrome. Fertil Steril. 2005, 84, 938-944.

20. La Marca A, Sunkara SK. Individualization of controlled ovarian stimulation in IVF using ovarian reserve markers: from theory to practice. Hum Reprod Update. 2014 20, 124-140.

21. Baarends W, Uilenbroek J, Kramer $P$, [et al.]. Anti-Mullerian hormone type II receptor maeesenger ribonucleotic acid expression in rat ovaries during postnatal development, the estrous cycle, and gonadotropin-induced follicle growth. Endocrinol. 1995, 136, 4951-4962.

22. Reichman DE, Goldschlag D, Rosenwaks Z. Value of antimüllerian hormone as a prognostic indicator of in vitro fertilization outcome. Fertil Steril. 2014 101, 1012.

23. Kedem A, Haas J, Geva LL, [et al.]. Ongoing pregnancy rates in women with low and extremely low AMH levels. A multivariate analysis of 769 cycles. PLoS One. 2013, 16, 8, e81629.

24. Łukaszuk K, Kunicki M, Liss J, [et al.]. Probability of live birth in women with extremely low anti-Müllerian hormone concentrations. Reprod Biomed Online. 2013, 28, 64-69.

25. Nelson SM, Yates RW, Lyall H, [et al.]. Anti-Müllerian hormone-based approach to controlled ovarian stimulation for assisted conception. Hum Reprod. 2009, 24, 867-875.

26. Brodin T, Hadziosmanovic N, Berglund L, [et al.]. Antimüllerian hormone levels are strongly associated with live-birth rates after assisted reproduction. J Clin Endocrinol Metab. 2013, 98, 1107-1114.
27. Rey RA, Sabourin J, Venara M, [et al.]. Anti-Müllerian hormone is specific marker of sertoli- and granulose-cell origin in gonadal tumors. Hum Pathol. 2000, 31, 1202-1203.

28. Stephen A, Pearsoll L, Christian B, [et al.]. Highly purified Müllerian inhibiting substance inhibits human ovarian cancer in vivo. Clin Cancer Res. 2002, 8, 2640-2646.

29. Peigné $M$, Decanter $C$. Serum AMH level as a marker of acute and long-term effects of chemotherapy on the ovarian follicular content: a systematic review. Reprod Biol Endocrinol. 2014, 26, 26.

30. Bersinger NA, Wunder D, Birkhauser MH, [et al.]. Measurement of anti-müllerian hormone by Beckman Coulter ELISA and DSL ELISA in assisted reproduction: differences between serum and follicular fluid. Clin Chim Acta. 2007, 384, 174-175.

31. Wallace $A M$, Faye $S A$, Fleming $R$, [et al.]. A multicentre evaluation of the new Beckman Coulter anti-Mullerian hormone immunoassay (AMH Gen II). Ann Clin Biochem. 2011, 48, 370-373.

32. Rustamov O, Smith A, Roberts SA, [et al.]. Anti-Mullerian hormone: poor assay reproducibility in a large cohort of subjects suggests sample instability. Hum Reprod. 2012, 27, 3085-3091.

33. Łukaszuk K, Ludwikowska B, Liss J, [et al]. Decreasing quality of the new generations of anti-Müllerian hormone assays. Biomed Res Int. 2014, 2014, 165352.

34. Su HI, Sammel MD, Homer MV, [et al.]. Comparability of antimüllerian hormone levels among commercially available immunoassays. Fertil Steril. 2014, 101, 1766-1772.

35. Gassner D, Jung R. First fully automated immunoassay for anti-Müllerian hormone. Clin Chem Lab Med. 2014, 52, 1143-1152. 